\title{
Research Square \\ Coherently amplifying photon production from vacuum with a dense cloud of accelerating photodetectors
}

Hui Wang ( $\sim$ hui.wang-2.gr@dartmouth.edu )

Dartmouth College https://orcid.org/0000-0003-1012-1124

Miles Blencowe

Dartmouth College https://orcid.org/0000-0003-0051-0492

\section{Article}

Keywords: photodetectors, vacuum photon production

Posted Date: February 4th, 2021

DOI: https://doi.org/10.21203/rs.3.rs-135207/v1

License: (c) (i) This work is licensed under a Creative Commons Attribution 4.0 International License. Read Full License

Version of Record: A version of this preprint was published at Communications Physics on June 10th, 2021. See the published version at https://doi.org/10.1038/s42005-021-00622-3. 


\title{
Coherently amplifying photon production from vacuum with a dense cloud of accelerating photodetectors
}

\author{
Hui Wang $^{1}$ and Miles Blencowe ${ }^{1}$ \\ ${ }^{1}$ Department of Physics and Astronomy, Dartmouth College, Hanover, New Hampshire 03755, USA
}

\begin{abstract}
We consider $N \gg 1$ accelerating (i.e., oscillating) photodetectors modeled as two level systems (TLSs) that are contained within a microwave cavity and show that the resulting photon production from vacuum can be collectively enhanced such as to be measurable. The cavity-accelerating TLSs system maps onto a parametrically driven Dicke-type model and when the detector number $N$ exceeds a certain critical value, the vacuum photon production undergoes a phase transition from a normal phase to an enhanced superradiant-like, inverted lasing phase. Such a model may be realized as a mechanical membrane with a dense concentration of optically active defects undergoing $\mathrm{GHz}$ flexural motion and contained within a 3D, superconducting microwave cavity. We show that recent related experimental devices are close to demonstrating this inverted, vacuum photon lasing phase.
\end{abstract}

\section{INTRODUCTION}

One of the most striking consequences of the interplay between relativity and the uncertainty principle is the predicted detection of real photons from the quantum electromagnetic field vacuum by non-inertial, accelerating photodetectors. For the special case of a photodetector with uniform, linear acceleration $a$ in Minkowski vacuum, the Unruh effect predicts that the detected photons are furthermore in a thermal state with temperature $T=\hbar a /\left(2 \pi c k_{B}\right)$ [1-5]. However, to measure for example a $1 \mathrm{~K}$ thermal photon temperature, a uniform detector acceleration $a=2.47 \times 10^{20} \mathrm{~m} / \mathrm{s}^{2}$ is required, which seems impossibly high for any current or planned tabletop experiments.

Given the difficulty in achieving uniform photodetector accelerations of sufficient magnitude and duration, certain experimental approaches have instead considered electrons that are accelerated nonuniformly by intense electromagnetic fields such that the trajectories are confined to a finite spatial volume. In particular, Bell and Leinass [6] interpreted the observed spin depolarization of electron(s) undergoing uniform circular motion in a storage ring in terms of 'heating' by vacuum fluctuations (see also Refs. [7, 8]). And Schutzhold et al. [9] showed that oscillating electrons emit both classical (Larmor) and quantum radiation, with the latter corresponding to the accelerating electrons converting quantum vacuum fluctuations into entangled photon pairs.

Motivated by the goal to enhance the detection of photons from vacuum, a proposal by Scully et al. [10] locates an accelerating photodetector atom within an electromagnetic cavity; the idealized, perfectly conducting walls of the enclosing cavity effectively modify the spacetime geometry from Minkowki space to a finite volume for the electromagnetic vacuum [2]. This finite confining cavity volume then necessitates a non-uniform, i.e., oscillatory acceleration, similar to the accelerating electron proposal of Ref. [9]; the photodetector atom is envisaged for example as attached to a piezoelectrically driven, vibrating cantilever structure. Another recent relevant proposal [11] considers a rapidly rotating, initially excited atom in a cavity, such that the atom's spontaneous emission is enhanced when sum of the rotation and atom splitting frequencies resonantly matches a cavity mode frequency.

In these investigations, the electrons and atom photodetectors accelerate nonuniformly (in either direction or magnitude) and in contrast to the Unruh effect as conventionally defined [3-5], the predicted photon spectrum is not expressible as a thermal distribution with temperature given solely in terms of the acceleration and fundamental constants $[12,13]$. Nevertheless, the entangled photon pair production and photon detection from the vacuum effects are still direct consequences of the non-inertial motion of electrons and atoms in quantum vacuum. 


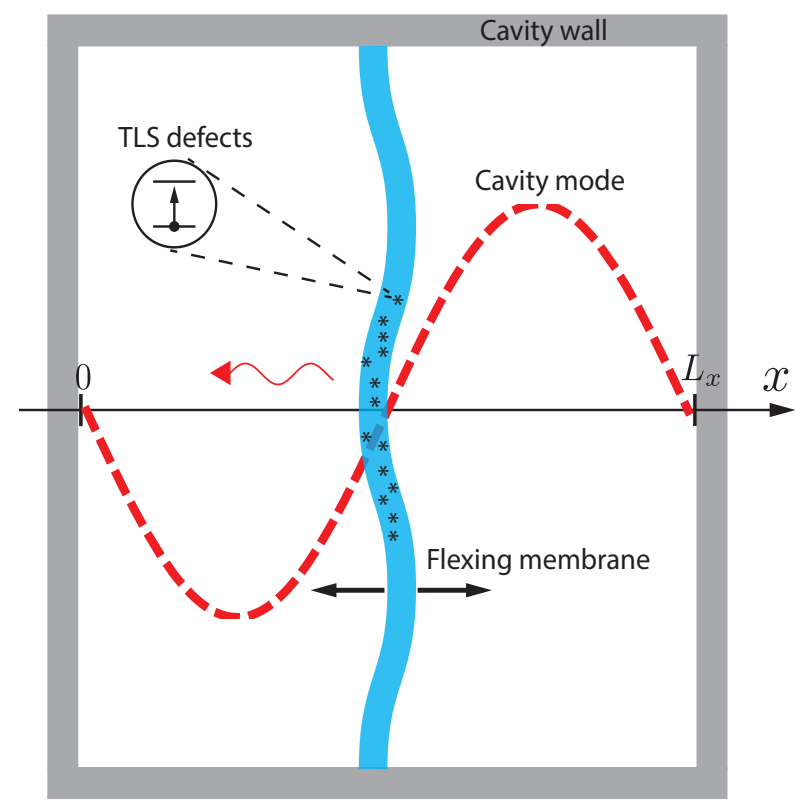

FIG. 1: Acceleration photodetection from vacuum scheme shown in crossection. A two-dimensional membrane located at $x=L_{x} / 2$, midway between cavity wall (mirror) boundaries at $x=0, L_{x}$, undergoes flexural vibrations normal to its surface in the $x$-coordinate direction, resulting in the oscillatory acceleration of pointlike, TLS photodetectors embedded within the membrane. An accelerating TLS can undergo a transition to its excited state level, with the accompanying emission of a photon into the cavity mode vacuum. A possible implementation might utilize a diamond membrane with nitrogen vacancy (NV) defect centers $[20,23,24]$ furnishing the TLSs, where the flexural vibrations of the membrane are induced for example by piezoactuators (not shown).

In recent work $[14,15]$, we proposed close analogs of the vacuum, oscillatory photodetection effect that involve coplanar (i.e., 2D) superconducting microwave cavity circuit systems [16]. The photodetector is modelled alternatively as a harmonic oscillator [14] and as a qubit [15] that capacitively couple to the 2D cavity via a mechanically oscillating film bulk acoustic resonator (FBAR) [17]; by increasing the FBAR capacitor plate area, a strong detector-cavity coupling can be achieved. Furthermore, when the FBAR mechanical frequency matches the sum of the cavity mode and detector ground-first excited energy level splitting, the photon production rate from cavity vacuum is resonantly enhanced [10] so as to be detectable. The use of an FBAR introduces an actual mechanical acceleration, in contrast to previous circuit-based analogs where the accelerating photodetector is mimicked by an electromagnetically induced, time changing detector coupling $[18,19]$.

However, a feasible way to demonstrate genuine oscillatory acceleration detection and production of photons from vacuum, where the photodetectors are accelerating within a 3D electromagnetic cavity (as opposed to the above 2D analogues) is still lacking. Despite the resonant gain resulting from placing an accelerating atom inside a cavity $[10,11]$, currently achievable cavity quality factors, atom-cavity coupling strengths, and mechanical oscillation acceleration magnitudes are insufficient for attaining measurable photon production from vacuum for a single accelerating detector.

In this paper, we describe a way to realize acceleration photodetection from vacuum with a scheme that 
involves many oscillating photodetectors contained within a microwave cavity (Fig. 1). Such a scheme may be realized by exploiting optically active defects that are embedded within a vibrating membrane structure, such that the defects effectively function as accelerating photodetectors. As one concrete example, we consider a $\mathrm{GHz}$ vibrating diamond membrane containing nitrogen vacancy (NV) color center defects [20]. We shall take advantage of a Dicke superradiance-like [21-24], vacuum amplification effect due to the collective motion of the many accelerating photodetectors. While the possibility has been mentioned before to collectively enhance photon production from vacuum using many accelerating electrons [9] or detectors [25, 26], no such investigations have been carried out previously to our knowledge. Note, however, the proposal of Ref. [27] to amplify and hence detect cavity photons produced via the dynamical Casimir effect (for an accelerating cavity mirror) through their superradiant-enhanced interaction with a cloud of optically pumped atoms. In Sec. II, we introduce our model for a cavity field interacting with $N \gg 1$ accelerating two level system (TLS) photodetectors and show how the model maps by approximation onto a simpler, parametrically driven Dicketype Hamiltonian [28]. Section III presents numerical and approximate analytical solutions to the model for various sample parameters, establishing the accuracy of the Dicke Hamiltonian description, as well as the existence of a phase transition in the vacuum photon production to a superradiant-like (properly termed 'inverted lasing') phase. In Sec. IV, we discuss the possible realization involving a GHz oscillating diamond membrane containing NV center defects $[20,23,24]$ functioning as TLS photodetectors enclosed within a 3D microwave cavity, and then give some concluding remarks.

\section{CAVITY- $N$ ACCELERATING DETECTORS MODEL}

Referring to Fig. 1, the $N$ TLS detectors are assumed to be embedded within a two-dimensional membrane material system undergoing driven, small $\mathrm{GHz}$ flexural vibrations in the $x$-coordinate direction (i.e., displacements normal to the static, equilibrium membrane $y-z$ surface), hence imparting acceleratory motion to the TLSs' centers of mass; the GHz membrane flexural motion may be actuated via piezoelectric transducers, for example. The membrane is enclosed with a cavity 'box' with volume $V=L_{x} L_{y} L_{z}$. For a microwave cavity, the box dimensions $L_{i}$ are of the order a few $\mathrm{cm}$. The cavity length in the flexural motion ( $x$ coordinate) direction is denoted as $L_{x}$, and the membrane is assumed to be located at the cavity midway point $x=L_{x} / 2$. We consider the following starting model action for the combined cavity field- $N$ detector system:

$$
\begin{aligned}
S= & -\int_{V} d^{3+1} x \frac{1}{2} \partial_{\mu} \Phi \partial^{\mu} \Phi+\sum_{i=1}^{N} \int d \tau_{i}\left\{\frac{m_{0}}{2}\left[\left(\partial_{\tau_{i}} Q_{i}\right)^{2}-\omega_{d 0}^{2} Q_{i}^{2}\right]-\frac{g}{4 !} Q_{i}^{4}\right\} \\
& +\lambda_{0} \sum_{i=1}^{N} \int_{V} d^{3+1} x Q_{i}\left(\tau_{i}\right) \Phi(t, \vec{r}) \delta^{3+1}\left[x^{\mu}-z_{i}^{\mu}\left(\tau_{i}\right)\right],
\end{aligned}
$$

where the detectors, classically modelled by internal, one dimensional anharmonic oscillator coordinates $Q_{i}, i=1, \ldots N$, are linearly coupled to a $3+1 \mathrm{D}$ massless scalar field denoted as $\Phi(t, \vec{r})$ that models the electromagnetic field within the cavity. Equation (1) generalizes the starting action of Ref. [29] to include more than one detector, as well as assumed weakly anharmonic oscillator potential energy terms $g Q_{i}^{4} / 4$ !. The latter allows the detectors' internal degrees of freedom to be truncated to TLSs under parametric resonance conditions; while the harmonic oscillator detector model is beneficial for analytical calculations [29], it is somewhat artificial since no detector is purely harmonic, and in particular results in an unphysical parametric instability beyond a critical field-detector(s) coupling [14]. The detectors are assumed to have identical center of mass rest frame internal harmonic oscillator frequencies $\omega_{d 0}$ and coupling strength $\lambda_{0}$ between each detector's internal degree of freedom and the cavity field. With the membrane undergoing driven flexural oscillations at some frequency $\Omega_{m}$, the center of mass of the $i$ th detector follows the worldline 
$z_{i}^{\mu}(t)=\left(t, L_{x} / 2+A \cos \left(\Omega_{m} t+\phi_{i}\right), y_{i}, z_{i}\right)$, where $A$ is the detector's center of mass acceleration amplitude and $\phi_{i}$ its phase. The phase $\phi_{i}$ accounts for the fact that the membrane's GHz flexural mode wavelength $(\sim$ few $\mu \mathrm{m})$ is much smaller than the extent of the detector distribution ( $\gtrsim$ few $\mathrm{mm})$, so that individual oscillating detectors may be out of phase with respect to each other depending on their relative separation in $y-z$ plane. The $i$ th detector's proper time is denoted by $\tau_{i}$, and its transverse center of mass coordinates are assumed to satisfy $y_{i} \sim L_{y} / 2$ and $z_{i} \sim L_{z} / 2$. The latter condition allows the simplifying approximation that the transversely distributed detectors couple equally to the resonant cavity mode. Note that we adopt the Minkowski metric sign convention $\eta_{\mu \nu}=\operatorname{diag}(-1,1,1,1)$.

The action (1) yields the following model Hamiltonian for the cavity-TLS detectors system in the rest frame of the cavity [30]:

$$
H=\hbar \omega_{c} a^{\dagger} a+\hbar \tilde{\omega}_{d} \sum_{i=1}^{N} \frac{d \tau_{i}}{d t} \frac{\sigma_{i}^{z}}{2}+\hbar \tilde{\lambda} \sum_{i=1}^{N} \frac{d \tau_{i}}{d t} \sin \left[k_{c} A \cos \left(\Omega_{m} t+\phi_{i}\right)\right]\left(a+a^{\dagger}\right) \sigma_{i}^{x},
$$

where we assume a single mode approximation for the cavity field with mode frequency $\omega_{c}=c k_{c}, k_{c}=$ $\sqrt{\left(2 \pi / L_{x}\right)^{2}+\left(\pi / L_{y}\right)^{2}+\left(\pi / L_{z}\right)^{2}}$, and we truncate the anharmonic oscillator detector state space to that spanned by the ground and first excited energy eigenstates with transition frequency $\tilde{\omega}_{d}$ and with TLS-cavity mode coupling $\tilde{\lambda}$ [30]. Here, we consider the $\left(n_{x}, n_{y}, n_{z}\right)=(2,1,1)$ cavity mode, which has a node at $L_{x} / 2$ (see Fig. 1) resulting in a first order dependence on oscillation amplitude $A$ in the interaction term of Hamiltonian (2); if we were to instead use the $\left(n_{x}, n_{y}, n_{z}\right)=(1,1,1)$ mode, the coupling would instead involve a 'cosine' instead of a 'sine' term and hence would be much smaller at second order in $A$. The cavity single mode approximation and detector two level truncation are justified under the condition of parametric resonance between the driven, flexural membrane frequency $\Omega_{m}$ and the (renormalized) detector and cavity mode frequencies (see Sec. III below). Hamiltonian (2) accounts for relativistic time dilation for the accelerating TLSs through the presence of the reciprocal Lorentz factors $d \tau_{i} / d t=\sqrt{1-\xi^{2} \sin ^{2}\left(\Omega_{m} t+\phi_{i}\right)}$, where $\xi=$ $\Omega_{m} A / c$, and may be thought of as a relativistic, parametrically driven Dicke model [28, 31].

With for example an achievable membrane flexural, microwave scale frequency $\Omega_{m} \sim 2 \pi \times 10 \mathrm{GHz}$ and oscillation amplitude $A \sim 10^{-10} \mathrm{~m}$, we have $\xi=\Omega_{m} A / c \sim 10^{-8}$ and hence the time dilation factors can be safely neglected for potential laboratory realizations. The sinusoidal interaction term can then be wellapproximated as $\sin \left[k_{c} A \cos \left(\Omega_{m} t+\phi_{i}\right)\right] \approx k_{c} A \cos \left(\Omega_{m} t+\phi_{i}\right)=\omega_{c} A / c \cos \left(\Omega_{m} t+\phi_{i}\right)$, and with $\omega_{c} \sim \Omega_{m}$ under conditions of resonance, we see that the individual TLS-cavity coupling $\tilde{\lambda}$ is effectively reduced by the maximum detector velocity-to-speed of light ratio $v_{m} / c \ll 1$.

Nevertheless, from a theoretical standpoint it is still interesting to allow also for relativistic TLS accelerations in exploring photon production when starting initially with the TLSs in their ground states and the cavity in its vacuum state. As we show just below, even under conditions of extreme relativistic detector motion, Hamiltonian (2) can be mapped onto a much simpler, time-independent Hamiltonian through a type of renormalized, rotating wave approximation (RWA) [30], provided the TLS-cavity coupling $\tilde{\lambda}$ is sufficiently small.

In particular, setting the phases $\phi_{i}=0$, we can describe the collection of $N$ TLSs as a single $N+1$-level system viewed as a large pseudospin vector of length $j=N / 2$, with the collective spin operators given by $J^{z} \equiv$ $\sum_{i=1}^{N} \sigma_{i}^{z} / 2$ and $J^{ \pm} \equiv \sum_{i=1}^{N} \sigma_{i}^{ \pm}$. Expanding the time-dependent terms in Eq. (2) and keeping only terms up to second harmonics in $\Omega_{m}$, the TLS transition frequency is renormalized as $\omega_{d}=\tilde{\omega}_{d} D_{0}$ and there is an additive frequency modulation $\tilde{\omega}_{d} D_{2} \cos \left(2 \Omega_{m} t\right)$, where $D_{0}$ and $D_{2}$ are $\xi$-dependent constants [30]. Imposing the resonance condition $\Omega_{m}=\omega_{c}+\omega_{d}$, we then transform the frequency renormalized, modulated Hamiltonian to the rotating frame via the unitary $U_{\mathrm{RF}}(t)=\exp \left(i \omega_{c} a^{\dagger} a t+i J^{z}\left[\omega_{d} t+\tilde{\omega}_{d} D_{2} \sin \left(2 \Omega_{m} t\right) / 2 \Omega_{m}\right]\right)$. Applying the rotating wave approximation (RWA), we finally have that the relativistic, driven Dicke type Hamiltonian (2) can be replaced by the following much simpler approximate Hamiltonian:

$$
H \approx \hbar \lambda\left(a^{\dagger} J^{+}+a J^{-}\right)
$$


where the coupling constant is $\lambda=\frac{1}{2} \tilde{\lambda} C_{1}\left[J_{0}\left(\tilde{\omega}_{d} D_{2} / 2 \Omega_{m}\right)-J_{1}\left(\tilde{\omega}_{d} D_{2} / 2 \Omega_{m}\right)\right]$, with $C_{1}$ a $\xi, \Omega_{m}$-dependent constant [30], and $J_{0}(z), J_{1}(z)$ are Bessel functions of the first kind.

An actual microwave cavity will be lossy, while TLSs in their excited state will relax through photon and phonon emission, with the latter decay channel a consequence of the TLSs embedded within an elastic membrane. For the potential realization discussed in Sec. IV, the dominant TLS relaxation channel is through phonon emission [23, 24], and since as mentioned above the phonon wavelength is much smaller than the extent of the TLS distribution, we model the TLSs as coupled to approximately independent environments. We assume that the cavity-TLSs system dynamics can be described by the following Lindblad master equation:

$$
\dot{\rho}=-\frac{i}{\hbar}[H, \rho]+\gamma_{c} \mathcal{L}_{a}[\rho]+\gamma_{d} \sum_{i=1}^{N} \mathcal{L}_{\sigma_{i}^{-}}[\rho]
$$

where $\gamma_{c}$ and $\gamma_{d}$ are the cavity and individual TLS energy damping rates, respectively, and the Lindblad superoperator is defined as $\mathcal{L}_{A}[\rho] \equiv A \rho A^{\dagger}-\frac{1}{2} A^{\dagger} A \rho-\frac{1}{2} \rho A^{\dagger} A$. Here, we suppose that the environment temperature is small compared to the frequencies of the cavity mode and TLSs (i.e., $k_{B} T / \hbar \ll \omega_{c}, \omega_{d}$ ), and also that each TLS has approximately the same damping rate; for the possible scheme described below in Sec. IV with microwave cavity and detector frequencies of a few $\mathrm{GHz}$, it suffices to work at dilution fridge temperatures of a few tens of $\mathrm{mK}$ or below in order to be in this low temperature regime.

\section{SOLUTIONS}

The single cavity mode approximation in Hamiltonian (2) is justified provided the approximate resonance condition $\Omega_{m} \approx \omega_{c}+\omega_{d}$ is satisfied [14]. This resonance condition also justifies the RWA Hamiltonian (3), provided $|\lambda| \ll \omega_{c}, \omega_{d}$. The advantage to working with the much simpler Hamiltonian (3) is that the Lindblad master equation (4) can be solved numerically for up to 20 or so TLSs, while for the full, relativistic time-dependent Hamiltonian (2), numerically solving the Lindblad equation is only possible for less than 10 TLSs before the computational run times become excessively long. It is noteworthy in this respect that using the approximate Hamiltonian (3) in the master equation provides an accurate description of the average photon number dynamics even for relativistic TLS center of mass motions with $\xi \lesssim 1$ [30]. Furthermore, for the assumed independent TLS damping, the average photon number dynamics is relatively insensitive to the phase $\phi_{i}$-dependencies of the TLSs' motions, justifying setting $\phi_{i}=0$ above [30]. From now on, we will use Hamiltonian (3) in the master equation (4).

One approach to solving this master equation begins with the Holstein-Primakoff (H-P) transformation $J^{+}=b^{\dagger} \sqrt{2 j-b^{\dagger} b}, J^{-}=\sqrt{2 j-b^{\dagger} b}$, which maps the collective spin operators $J^{ \pm}$to the bosonic creation and annihilation operators $b^{\dagger}, b[32]$. In the large $j=N / 2$ limit, the collective spin of the $N$ TLSs is approximated as a single harmonic oscillator, with Hamiltonian (3) mapped to $\hbar \sqrt{N} \lambda\left(a^{\dagger} b^{\dagger}+a b\right)$. This coincides with the RWA Hamiltonian that was used in Ref. [14] to describe the oscillatory acceleration for a system comprising a photodetector coupled to a cavity electromagnetic field in the single mode approximation, with the detector's internal degrees of freedom modeled as a harmonic oscillator, and the detector's center of mass oscillating at a frequency $\Omega_{m}$ that matches the sum of the cavity frequency and detector internal frequency. This system describes a nondegenerate parametric amplification process, with cavity-detector photon pairs produced from the vacuum via mechanical pumping. Including cavity and detector damping and solving analytically the corresponding master equation for the above bosonic Hamiltonian, the average cavity photon number in the long time limit is $\left\langle a^{\dagger} a\right\rangle=4 \gamma_{d} N \lambda^{2} /\left[\left(\gamma_{c}+\gamma_{d}\right)\left(\gamma_{c} \gamma_{d}-4 N \lambda^{2}\right)\right]$. Note that the system exhibits a parametric instability when the effective coupling strength $\sqrt{N} \lambda$ exceeds the value $\sqrt{\gamma_{c} \gamma_{d}} / 2$. While such an instability indicates a breakdown of the above H-P derived approximation method, it does point to the 
existence of an enhanced vacuum photon production phase for $N>N_{\text {crit }}=\gamma_{c} \gamma_{d} /\left(4 \lambda^{2}\right)$ in the original cavity-TLSs model dynamics given by equations (2-4), where there is no such instability.
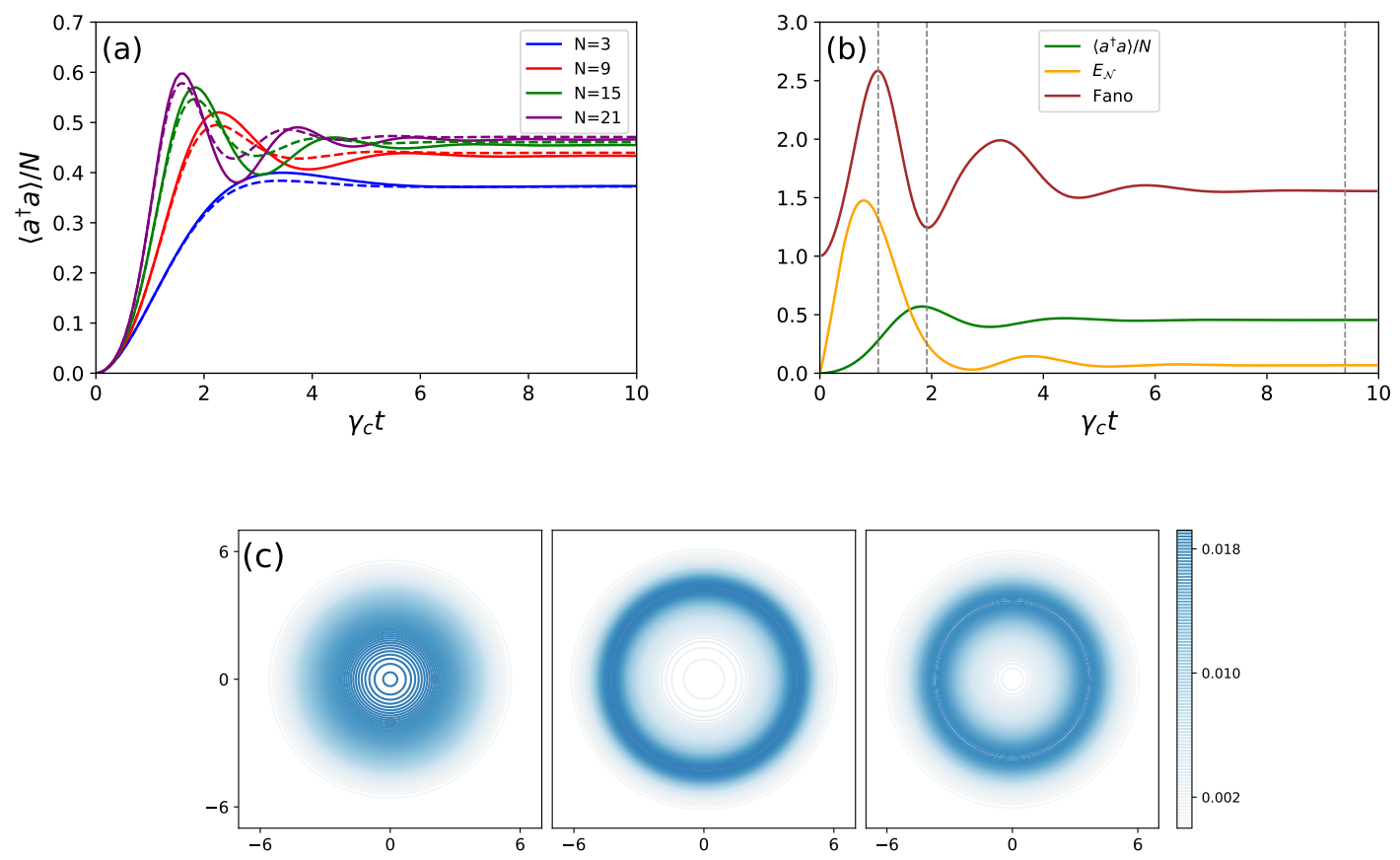

FIG. 2: (a) Dynamical evolution of the scaled cavity average photon number: $\left\langle a^{\dagger} a\right\rangle / N$; solid lines are the numerical solutions and dashed lines are the cumulant expansion approximation. (b) Evolution of $\left\langle a^{\dagger} a\right\rangle / N$, Fano factor, as well as the entanglement (logarithmic negativity $E_{\mathcal{N}}$ ) for $N=15$ case; the vertical dashed lines indicate the approximate time instants corresponding to the Wigner function snapshots appearing in (c). The parameters are $\gamma_{c}=\gamma_{d}=0.02$ and $\lambda=0.01$ (in units $\omega_{c}=1$ ), corresponding to $N_{\text {crit }}=1$.

In Fig. 2a, we show for some example, illustrative parameters corresponding to $N_{\text {crit }}=1$, the dynamical behavior of the average scaled cavity photon number $\left\langle a^{\dagger} a\right\rangle / N$ starting from the cavity mode vacuum and with all the TLSs initially in their ground states at time $t=0$. The solid line plots are obtained by numerically solving [33] the Lindblad master equation (4) with the RWA Hamiltonian (3); the utilized numerical method exploits the permutation symmetry of the density operator [34], which reduces the size of the Hilbert space required for the TLSs and increases the accessible value of $N$ up to around 20, depending on the parameter choices. The dashed line plots are obtained by solving approximate equations for the non-vanishing first and second order moments derived from the master equation (4). We find dramatically improved accuracy as compared with usual cumulant expansion approximation methods [35, 36], even for relatively small $N$, by instead setting certain fourth order instead of third order cumulants to zero [30]. In particular, with each of the TLSs giving an identical contribution to the moment equations, we can replace $\sigma_{i}^{z}, \sigma_{i}^{ \pm} \sigma_{j}^{z}(i \neq j)$ with $\sigma_{1}^{z}$, $\sigma_{2}^{ \pm} \sigma_{1}^{z}$ respectively. Furthermore, utilizing the identity $\sigma_{1}^{z}=2 \sigma_{1}^{+} \sigma_{1}^{-}-1$ and approximating that the fourth cumulants vanish, we obtain for example the following non-vanishing third moment approximation [30]: $\left\langle a^{\dagger} \sigma_{2}^{+} \sigma_{1}^{z}\right\rangle=\left\langle a^{\dagger} \sigma_{2}^{+}\right\rangle\left\langle\sigma_{1}^{z}\right\rangle+2\left\langle a^{\dagger} \sigma_{1}^{+}\right\rangle\left\langle\sigma_{1}^{-} \sigma_{2}^{+}\right\rangle$. Note that the latter approximation contains an additional term involving products of second moments as compared with the usually employed, third cumulant vanishing 
approximation $[35,36]$.

The purpose for using the above cumulant expansion approximation is that the photon production from cavity vacuum can be investigated for $N \ggg 1$, relevant for potential realizations (see Sec. IV), while the numerical Lindblad solutions for smaller $N$ are useful for validating the cumulant approximation, as well as giving a more complete picture of the quantum dynamics.

As $N$ increases above $N_{\text {crit }}$, a 'burst' peak of cavity photon production from vacuum appears in Fig. 2a that progressively grows in magnitude, narrows, and shifts to earlier times. Furthermore, the long time limit steady state average photon number grows in magnitude. These features qualitatively resemble those of Dicke superradiance $[21,22]$, although in the latter process the TLSs are initially prepared in their excited state and the cavity in the ground state. (Note that Ref. [37] considers the effect on superradiance of $N=6$ uniformly accelerating, initially excited photodetectors, which is different from the collective enhancement effect for photon detection from vacuum considered here). In order to get a better idea of the cavity mode-TLSs state, in Fig. 2b we show for $N=15$ the time dependence of the logarithmic negativity entanglement measure $E_{\mathcal{N}}$ [38] between the cavity and TLS ensemble, and also the cavity Fano factor $\left(\left\langle\left(a^{\dagger} a\right)^{2}\right\rangle-\left\langle a^{\dagger} a\right\rangle^{2}\right) /\left\langle a^{\dagger} a\right\rangle$ in comparison with the average scaled cavity photon number. The entanglement grows and reaches a maximum roughly when the Fano factor is a maximum, while the entanglement is relatively small, although non-zero, in the long-time limit. This non-zero entanglement growth from vacuum indicates that the cavity photon production and TLSs' excitation is correlated. The Fano factor provides a more quantitative measure of the photon number variance than the Wigner function snapshots shown in Fig. 2c; these latter snapshots correspond to the instants when the Fano factor reaches its peak, subsequent trough, and long-time limit steady state. The photon production from vacuum burst corresponds to the cavity mode Wigner function rapidly first spreading out and then forming a ring with width close to that of a coherent state, and eventually settling into a thicker ring in the steady state due to environmental diffusion.

\section{DISCUSSION}

In order to get a more quantitative sense of the average cavity photon number dynamics scaling dependence on TLS number $N$, as well as model possible experimental set-ups, we must go to the large $N_{\text {crit }}$ limit where it is not feasible to numerically solve the master equation (4). As an alternative, we can apply the approximate second order moment equations [30] that should become increasingly accurate in the large $N$ limit, provided $N$ is not too close to $N_{\text {crit }}[35,36]$, and which can be straightforwardly solved numerically for arbitrarily large $N$ since the number of coupled moment equations is fixed and small.

We will assume in part the parameters of the 3D microwave cavity-coupled nitrogen vacancy (NV) center defect scheme of Refs. [23, 24], which observed signatures of Dicke superradiance [21, 22]. In particular, we consider a coupling strength $\tilde{\lambda}=2 \pi \times 70 \mathrm{mHz}$, corresponding to an $\mathrm{NV}$ defect coupling via its magnetic moment to the magnetic field component of the considered cavity electromagnetic vacuum mode with frequency $\omega_{c}=2 \pi \times 3.2 \mathrm{GHz}$ [24]. Assuming a diamond membrane flexural oscillation amplitude $A=10^{-10} \mathrm{~m}$, the nonrelativistic coupling strength is $\lambda=\tilde{\lambda} \omega_{c} A /(2 c) \approx 1.5 \times 10^{-9} \mathrm{~Hz}$. For the NV defects, the dominant relaxation process is through spin-phonon interactions with rate $\gamma_{d} \approx 2 \times 10^{-4} \mathrm{~Hz}[24]$. On the other hand, assuming a realizable, superconducting microwave cavity quality factor $Q_{c}=10^{6}$ [39], we have $\gamma_{c}=\omega_{c} / Q_{c} \approx 2 \times 10^{4} \mathrm{~Hz}$. With these numbers, we have $N_{\text {crit }}=\gamma_{c} \gamma_{d} /(2 \lambda)^{2} \approx 4 \times 10^{17}$; the number $N$ of microwave field-coupled defects in the experiment of Ref. [24] is in the range $(0.36-1.5) \times 10^{16}$, not far below this $N_{\text {crit }}$ value.

Figure 3a gives the first burst peak maximum and the long time limit steady state rescaled cavity photon number $\left\langle a^{\dagger} a\right\rangle / N$ dependences on $N / N_{\text {crit }}$. We observe clear evidence of a phase transition, with the slope of average photon number dependence on $N$ changing sharply as $N$ moves through $N_{\text {crit }}$. In particular, in the long time steady state, we obtain the following approximate analytical expressions well below and above 

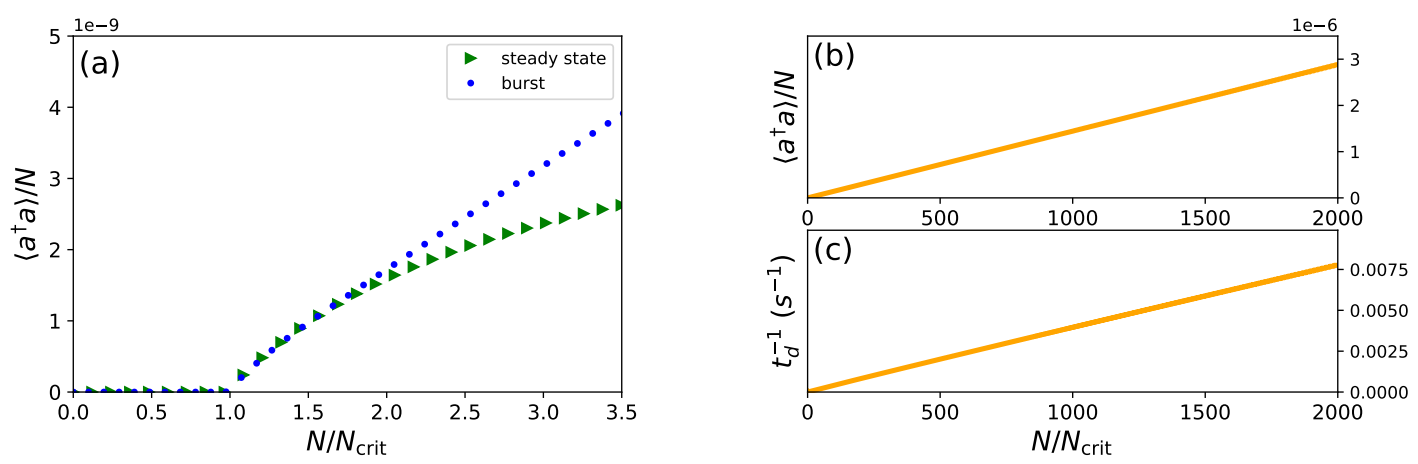

FIG. 3: (a) First vacuum photon production burst peak and long time limit steady state scaled cavity photon numbers $\left\langle a^{\dagger} a\right\rangle / N$ versus $N / N_{\text {crit }}$. (b) First peak value of $\left\langle a^{\dagger} a\right\rangle / N$ and (c) first peak inverse delay time $1 / t_{d}$ versus $N / N_{\text {crit }}$ for $N \gg N_{\text {crit }}$. Assumed parameters are $\gamma_{c} \approx 2 \times 10^{4} \mathrm{~Hz}, \gamma_{d} \approx 2 \times 10^{-4} \mathrm{~Hz}$, and $N_{\text {crit }} \approx 4 \times 10^{17}$.

$N_{\text {crit }}$, respectively [30]: $\left\langle a^{\dagger} a\right\rangle=\frac{N}{N_{\text {crit }}} \frac{\gamma_{d}}{\gamma_{c}+\gamma_{d}} \approx 2 \times 10^{-26} N \lll 1$ for $N \ll N_{\text {crit }}$, and $\left\langle a^{\dagger} a\right\rangle=N \gamma_{d} /\left(2 \gamma_{c}\right) \approx$ $5 \times 10^{-9} N \ggg 1$ for $N \gg N_{\text {crit }}$; we can clearly see that the average cavity photon number generated from vacuum is negligible below and non-negligible above $N_{\text {crit }}$. While these steady state average photon numbers scale linearly with $N$, from Fig. 3b we see that in contrast the first burst peak scales as $N^{\alpha}$ with $\alpha \approx 2$ for $N>N_{\text {crit }}$ (i.e., quadratically with $N$ to a good approximation); the peak average photon number rapidly grows in magnitude relative to the steady state value with increasing $N$ above $N_{\text {crit }}$. In Fig. 3c, we see that the delay $t_{d}$ in the appearance of this first burst peak starting from the initial cavity vacuum state becomes shorter as $N$ increases, with the inverse delay $t_{d}^{-1}$ scaling linearly with $N$.

While these observed scaling dependencies for the first burst peak of photon production from vacuum coincide with those for Dicke superradiant bursts [24], they are not properly a superradiant phase however [36]. In order to understand better the nature of this enhanced vacuum photon production phase, it is informative to apply the unitary transformation $U=\exp \left(i \pi J^{x} / 2\right)$ to the master equation (4); the RWA Hamiltonian (3) then transforms to the Tavis-Cummings Hamiltonian $H=\hbar \lambda\left(a^{\dagger} J^{-}+a J^{+}\right)$, which involves co-rotating terms only, while the Lindblad operators $\mathcal{L}_{\sigma_{i}^{-}}[\rho]$ are transformed to $\mathcal{L}_{\sigma_{i}^{+}}[\rho]$, with each TLS initial ground state transformed to its corresponding excited state. The cavity-oscillatory TLSs system is thus unitarily equivalent to a Tavis-Cummings model with an incoherent pump. While the latter model does not exhibit a Dicke superradiant transition, which requires the presence of both co-rotating and counterrotating terms of the Hamiltonian, it nevertheless exhibits a so-called lasing transition [36]. For this reason, the transition to enhanced acceleration detection from vacuum may be thought of as a transition from a normal, incoherent phase below $N_{\text {crit }}$ to a coherent inverted lasing phase above $N_{\text {crit }}$.

The challenge of any experimental scheme is to exceed the threshold for coherent photon production from vacuum (equivalently the inverted lasing threshold [36]), which in terms of the TLS number $N$ is given by the condition $N>N_{\text {crit }}=\gamma_{c} \gamma_{d} /(2 \lambda)^{2}$. In order to reduce the size of $N_{\text {crit }}$, we require microwave cavities with large quality factors, TLS's with small damping rates, and large TLS-photon coupling strengths $\lambda$. One way to enhance $\lambda$ is to reduce the microwave cavity volume, for example by utilizing $2 \mathrm{D}$ coplanar microwave cavities [40-42]. However, this creates challenges for locating a sufficiently large number of TLSs within the microwave cavity region. On the other hand, while achievable $\lambda$ couplings for 3D microwave cavities are a few orders of magnitude smaller than what is possible for $2 \mathrm{D}$ coplanar cavities, correspondingly much greater numbers of TLSs can be located within the 3D microwave cavity region, furnished by defects within a 
flexurally vibrating membrane. The above mentioned NV centre scheme for probing Dicke superradiance [23, $24]$ is a promising direction, although larger cavity quality factors are required, as well as larger surface area diamond membranes that can be actuated into $\mathrm{GHz}$ frequency flexural motion.

While we have focused in the present investigation on a microwave cavity-GHz oscillating membrane defect scheme, it is worth considering other possible schemes as well that effectively incorporate many accelerating photodetector/emitter degrees of freedom. In particular, it would be interesting to revisit the oscillating electron-photon pair production scheme of Ref. [9], but instead consider many electrons accelerating in a strong, periodic electromagnetic field. Under conditions where the dominant wavelength of emitted photon pairs is large compared to the average electron separation, we might expect a superradiant-like coherent enhancement of the pair production rate. Alternatively, we might consider a cloud of atoms (i.e., quantum dipoles) [43], although the challenge to impart sufficient acceleration magnitude to the cloud's center of mass would need to be addressed. Furthermore, electron-electron and dipole-dipole interactions would likely need to be taken into account; such interactions might result in quantum synchronization [43], where small initial differences in the individual electron/dipole acceleration magnitudes and phases do not influence the resulting coherent emission of radiation starting from the vacuum state. Finally, with the long-sought goal to demonstrate the original Unruh effect, it would be interesting to investigate possible collective enhancements of photodetection from vacuum for a dense cloud of detectors accelerating uniformly over a sufficiently long time interval in Minkowski vacuum, such that the detected photon spectrum is thermal to a good approximation.

In conclusion, we have proposed a way to demonstrate photon production from vacuum as a consequence of genuinely accelerating photodetectors. Our scheme involves a membrane with a dense cloud of embedded NV defects (equivalently TLS photodetectors) undergoing driven, transverse flexural vibrations within a microwave cavity, and modelled as a driven Dicke-type Hamiltonian. Under the condition of resonance, where the TLSs' center of mass acceleration frequency matches the sum of the cavity mode and internal TLSs' transition frequency, the system Hamiltonian considerably simplifies via the RWA, thus allowing for an accurate analytical solution using a cumulant expansion approach. When the number $N$ of TLS defects exceeds a critical value $N_{\text {crit }}$, the system undergoes a transition to an inverted lasing phase, signalled by a 'burst' peak in the average cavity photon number that scales as $N^{2}$, yielding significantly enhanced, collective photon production from vacuum. While the primary motivation for the present investigation concerns the interplay between the fundamental physics of relativistic quantum field vacuum and many body quantum dynamics, the possibility to realize entangled many TLS (i.e., qubit)-microwave photon states, purely by mechanically driving a membrane structure in cavity vacuum, may also find broader relevance and application in quantum information science and technology.

\section{Acknowledgements}

We thank A. D. Armour, W. F. Braasch, S.-Y. Lin, O. B. Wright, N. Shammah, A. R. H. Smith, and J. Yang for very helpful discussions. This work was supported by the NSF under grant nos. DMR-1507383 and PHY-2011382.

[1] W. Unruh, Phys. Rev. D 14, 870 (1976).

[2] B. S. DeWitt, General Relativity: An Einstein Centenary Survey, edited by S.W. Hawking and W. Israel (Cambridge University Press, Cambridge, U.K., 1980).

[3] L. C. B. Crispino, A. Higushi, G. E. A. Matsas, Rev. Mod. Phys. 80, 787 (2008).

[4] S. A. Fulling and G. E. Matsas, Scholarpedia 9, 31789 (2014). 
[5] J. S. Ben-Benjamin, M. O. Scully, S. A. Fulling, D. M. Lee, D. N. Page, A. A. Svidzinsky, M. S. Zubairy, M. J. Duff, R. Glauber, W. P. Schleich, and W. G. Unruh, Int. J. Mod. Phys. A 34, 1941005 (2019).

[6] J. S. Bell and J. M. Leinaas, Nucl. Phys. B 284, 488 (1987).

[7] W. G. Unruh, Phys. Rep. 307, 163 (1998).

[8] S. Biermann, S. Erne, C. Gooding, J. Louko, J. Schmiedmayer, W. G. Unruh, and S. Weinfurtner, Phys. Rev. D 102, 085006 (2020).

[9] R. Schützhold, G. Schaller, and D. Habs, Phys. Rev. Lett. 100, 091301 (2008).

[10] M. O. Scully, V. V. Kocharovsky, A. Belyanin, E. Fry, and F. Capasso, Phys. Rev. Lett. 91243004 (2003).

[11] K. Lochan, H. Ulbricht, A. Vinante, and S. K. Goyal, Phys. Rev. Lett. 125, 241301 (2020).

[12] B. L. Hu and A. Roura, Phys. Rev. Lett. 93, 129301 (2004).

[13] J. Doukas, S.-Y. Lin, B. L. Hu, and R. Mann, J. High Energy Phys. 11, 119 (2013).

[14] H. Wang, M. P. Blencowe, C. M. Wilson, and A. J. Rimberg, Phys. Rev. A 99, 053833 (2019).

[15] M. P. Blencowe, and H. Wang, Phil. Trans. R. Soc. A. 378, 20190224 (2020).

[16] P. D. Nation, J. R. Johansson, M. P. Blencowe, and F. Nori, Rev. Mod. Phys. 84, 1 (2012).

[17] M. Sanz, W. Wieczorek, S. Gröblacher, and E. Solano, Quantum 2, 91 (2018).

[18] S. Felicetti, C. Sabín, I. Fuentes, L. Lamata, G. Romero, and E. Solano, Phys. Rev. B 92, 064501 (2015).

[19] L. García-Álvarez, S. Felicetti, E. Rico, E. Solano, and C. Sabín, Sci. Rep. 7, 657 (2017).

[20] A. H. Piracha, K. Ganesan, D. W. M. Lau, A. Stacey, L. P. McGuinness, S. Tomljenovic-Hanic, and S. Prawer, Nanoscale 8, 6860 (2016).

[21] R. H. Dicke, Phys. Rev. 93, 99 (1954).

[22] M. Gross and S. Haroche, Phys. Rep. 93, 301 (1982).

[23] A. Angerer, T. Astner, D. Wirtitsch, H. Sumiya, S. Onoda, J. Isoya, S. Putz, and J. Majer, Appl. Phys. Lett. 109, 033508 (2016)

[24] A. Angerer, K. Streltsov, T. Astner, S. Putz, H. Sumiya, S. Onoda, J. Isoya, W. J. Munro, K. Nemoto, J. Schmiedmayer, and J. Majer, Nature Phys. 14, 1168 (2018).

[25] M. del Rey, D. Porras, and E. Martín-Martínez, Phys. Rev. A 85, 022511 (2012).

[26] M. del Rey, PhD thesis, Universidad Complutense de Madrid, (2015).

[27] W.-J. Kim, J. H. Brownell, and R. Onofrio, Phys. Rev. Lett. 96, 200402 (2006).

[28] R. Chitra, and O. Zilberberg, Phys. Rev. A 92, 023815 (2015).

[29] S.-Y. Lin and B. L. Hu, Phys. Rev. D 73, 124018 (2006).

[30] Supplementary Information.

[31] V. M. Bastidas, C. Emary, B. Regler, and T. Brandes, Phys. Rev. Lett. 108043003 (2012).

[32] T. Holstein and H. Primakoff, Phys. Rev. 58, 1098 (1949).

[33] J. R. Johansson, P. D. Nation, and F. Nori, Comput. Phys. Commun.184, 1234 (2013).

[34] N. Shammah, S. Ahmed, N. Lambert, S. DeLiberato, and F.Nori, Phys. Rev. A 98, 063815 (2018).

[35] P. Kirton and J. Keeling, Phys. Rev. Lett. 118, 123602 (2017).

[36] P. Kirton and J. Keeling, New J. Phys. 20, 015009 (2018).

[37] B. Richter, H. Terças, Y. Omar, and I. de Vega, Phys. Rev. A 96, 053612 (2017).

[38] G. Vidal and R. F. Werner, Phys. Rev. A 65, 032314 (2002).

[39] M. Stammeier, S. Garcia, and A. Wallraff, Quantum Sci. Technol. 3, 045007 (2018).

[40] M. Devoret, S. Girvin, and R. Schoelkopf, Ann. Phys. (Leipzig) 16, 767 (2007).

[41] S. Probst, A. Bienfait, P. Campagne-Ibarcq, J. J. Pla, B. Albanese, J. F. Da Silva Barbosa, T. Schenkel, D. Vion, D. Esteve, K. Mølmer, J. J. L. Morton, R. Heeres, and P. Bertet, Appl. Phys. Lett. 111, 202604 (2017).

[42] V. Ranjan, S. Probst, B. Albanese, T. Schenkel, D. Vion, D. Esteve, J. J. L. Morton, and P. Bertet, Appl. Phys. Lett. 116, 184002 (2020).

[43] B. Zhu, J. Schachenmayer, M. Xu, F. Herrera, J. G. Restrepo, M. J. Holland, and A. M. Rey, New J. Phys. 17, 083063 (2015). 


\section{Figures}

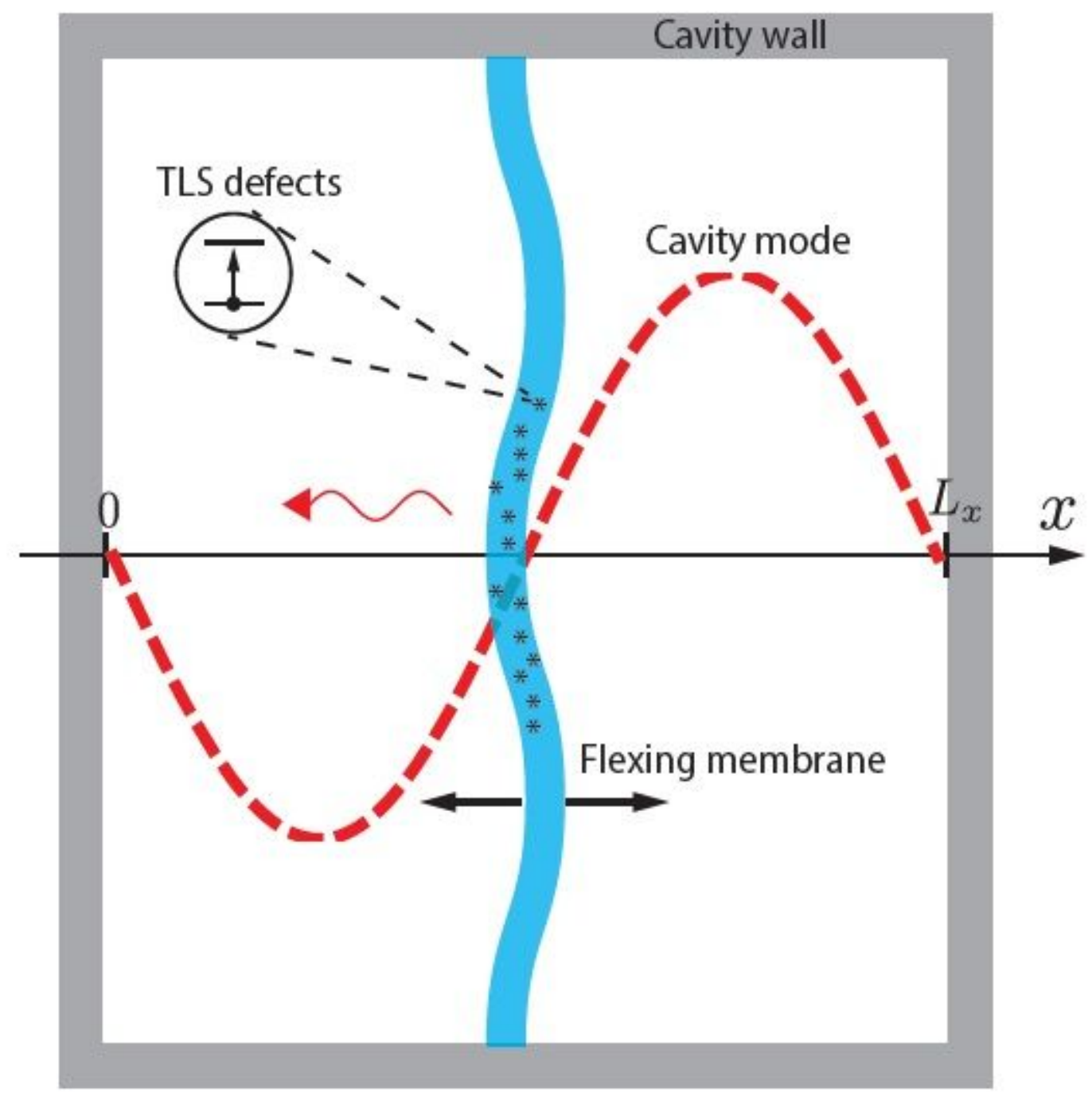

Figure 1

Acceleration photodetection from vacuum scheme shown in crossection. A two-dimensional membrane located at $x=L x=2$, midway between cavity wall (mirror) boundaries at $x=0 ; L x$, undergoes exural vibrations normal to its surface in the $x$-coordinate direction, resulting in the oscillatory acceleration of pointlike, TLS photodetectors embedded within the membrane. An accelerating TLS can undergo a transition to its excited state level, with the accompanying emission of a photon into the cavity mode vacuum. A possible implementation might utilize a diamond membrane with nitrogen vacancy (NV) defect centers $[20,23,24]$ furnishing the TLSs, where the exural vibrations of the membrane are induced for example by piezoactuators (not shown). 

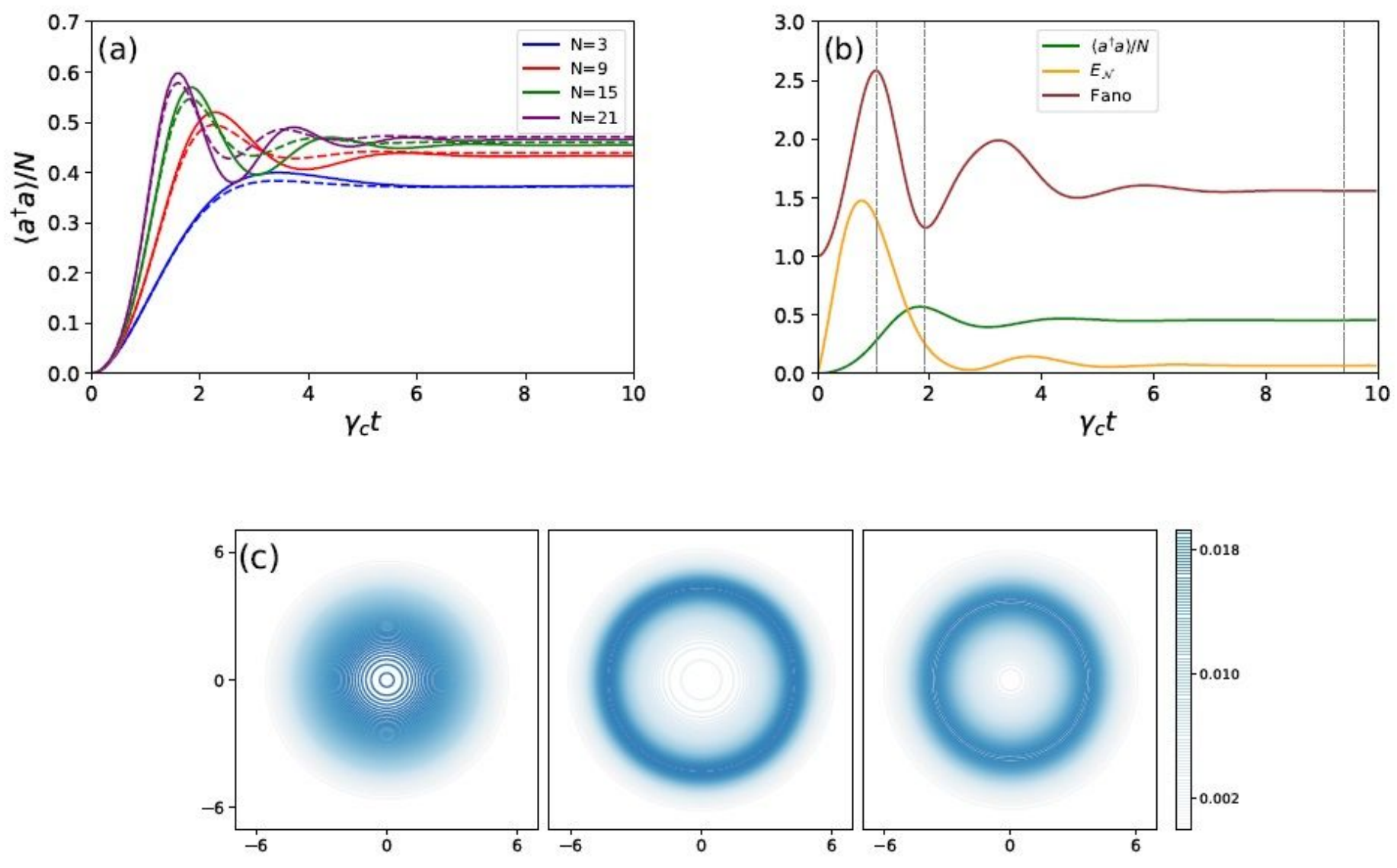

\section{Figure 2}

(a) Dynamical evolution of the scaled cavity average photon number: hayai=N; solid lines are the numerical solutions and dashed lines are the cumulant expansion approximation. (b) Evolution of hayai=N, Fano factor, as well as the entanglement (logarithmic negativity $\mathrm{EN}$ ) for $\mathrm{N}=15$ case; the vertical dashed lines indicate the approximate time instants corresponding to the Wigner function snapshots appearing in (c). The parameters are $c=d=0: 02$ and $x=0: 01$ (in units $! c=1$ ), corresponding to Ncrit $=1$.
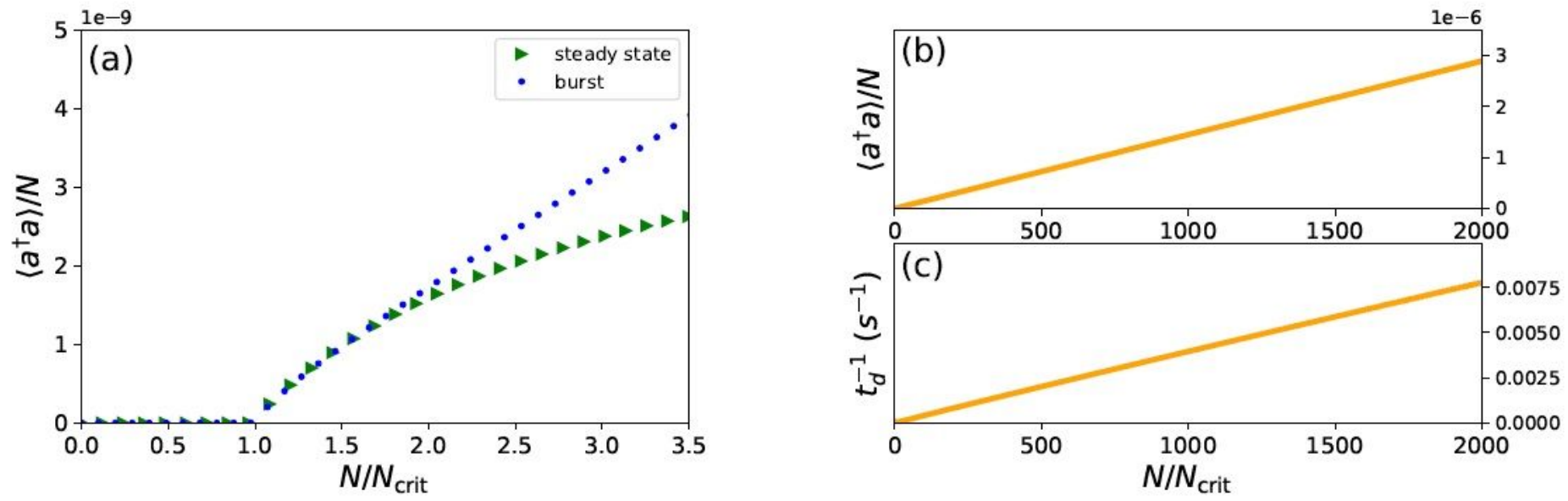

Figure 3 
(a) First vacuum photon production burst peak and long time limit steady state scaled cavity photon numbers hayai $=\mathrm{N}$ versus $\mathrm{N}=\mathrm{N}$ crit. (b) First peak value of hayai $=\mathrm{N}$ and (c) first peak inverse delay time $1=t d$ versus $\mathrm{N}=$ Ncrit for $\mathrm{N}$ >> Ncrit. Assumed parameters are c $\sim 2 \times 104 \mathrm{~Hz}, \mathrm{~d} \sim 2 \times 104 \mathrm{~Hz}$, and Ncrit $\sim 4$ x 1017.

\section{Supplementary Files}

This is a list of supplementary files associated with this preprint. Click to download.

- WangSM.pdf 